\title{
Programa de ensino de facoemulsificação CBO/ALCON: resultados do Hospital de Olhos do Paraná
}

\author{
CBO/ALCON teaching method of phacoemulsification: results of Hospital de Olhos do Paraná
}

Ana Flávia de Castro Fischer ${ }^{1}$, Eduardo Machado Estevão Pires $^{1}$, Fernando Klein ${ }^{2}$, Otávio Siqueira Bisneto ${ }^{1}$, Eduardo Sone Soriano ${ }^{3}$, HAMILTON MOREIRA ${ }^{4}$

\section{RESUMO}

Objetivo: Analisar os resultados obtidos com a aplicação do programa de ensino de facoemulsificação CBO/ALCON no curso de especialização em oftalmologia do Hospital de Olhos do Paraná.

Métodos: Realizou-se um estudo retrospectivo analítico dos resultados das cirurgias de catarata realizadas pelo programa de ensino de facoemulsificação CBO/ALCON que utiliza o método "reverso" em pacientes provenientes do ambulatório do SUS do Hospital de Olhos do Paraná. O programa consta da progressão dos alunos por cinco etapas chamadas de "check points", sendo analisadas as intercorrências per-operatórias em cada um deles, bem como as pós-operatórias.

Resultados: Do total de 84 olhos operados, $77(91,67 \%)$ apresentaram acuidade visual (AV) final de 20/40 ou melhor. Entre as intercorrências, a principal delas foi a rotura de cápsula posterior (RCP) e ocorreu em 11 (13,09\%) dos 13 $(15,48 \%)$ olhos que apresentaram algum tipo de complicação.

Conclusão: O método "reverso" proposto para o ensino de facoemulsificação pelo programa CBO/ALCON se mostra uma forma de ensino adequada, para o médico residente e seu instrutor, e principalmente para o paciente, devido ao baixo índice de complicações.

Descritores: Oftalmologia/educação; Facoemulsificação; Acuidade visual; Extração de catarata/efeitos adversos; Competência clínica; Aprendizagem

\begin{abstract}
Purpose: To analyze the results obtained with a new phacoemulsification teaching method in the specialization course of Hospital de Olhos do Paraná. Methods: A retrospective study evaluated the results of a "reversal" method used in the CBO/ALCON teaching program at the Hospital de Olhos do Paraná. The method is based on the student progression through five checkpoints starting from the end of the surgery towards the beginning and analysis of intra- and postoperative complications at each one of the checkpoints.

Results: Of 84 operated eyes, 77 (91.67\%) presented final visual acuity of 20/40 or better. Among the complications, the main one was the posterior capsular rupture and occurred in 11 (13.09\%) of 13 (15.48\%) eyes with complications. Conclusion: The "reversal" teatching method used in the CBO/ALCON teaching program is appropriate to the student, his instructor, as well as to the patient.
\end{abstract}

Keywords: Ophthalmology/education; Phacoemulsification; Visual acuity; Cataract extraction/adverse effects; Clinical competence; Learning

\section{INTRODUÇÃO}

A catarata é anatomicamente definida como qualquer opacificação do cristalino que difrate a luz, acarretando efeito negativo na visão, que pode variar desde uma pequena diminuição da acuidade visual até mesmo a cegueira. É a principal causa tratável de cegueira em todo o mundo ${ }^{(1-4)}$. Segundo a Organização Mundial de Saúde, há 45 milhões de cegos no mundo, dos quais $40 \%$ são devidos à catarata ${ }^{(5)}$.

Várias são as causas da catarata: trauma, associação com doenças sistêmicas ou uso de drogas, consequência de outras doenças e as possíveis desordens congênitas, sendo a senilidade a mais comum delas. Assim, estima-se que 10\% da popula-

Trabalho realizado no Hospital de Olhos do Paraná - Curitiba (PR), Brasil.

1 Médico, Hospital de Olhos do Paraná - Curitiba (PR), Brasil.

2 Aluno de Medicina, Universidade Positivo - Curitiba (PR), Brasil.

3 Médico, Instituto de Catarata - INCAT - Departamento de Oftalmologia, Universidade Federa de São Paulo - UNIFESP - São Paulo (SP) - Brasil.

${ }^{4}$ Médico, Hospital de Olhos do Paraná - Curitiba (PR) - Brasil; Universidade Federal do Paraná UFPR - Curitiba (PR) - Brasil.

Endereço para correspondência: Fernando Klein. Rua Dr. Faivre, 63 - Apto. 104 Curitiba (PR) - CEP 80060-140 - Email: klein80@gmail.com

Os autores não tem interesse comercial em nenhum dos produtos citados neste estudo.

Recebido para publicação em 31.03.2010

Última versão recebida em 24.08.2010

Aprovação em 24.10.2010 ção norte-americana têm catarata e que esta prevalência aumenta em 50\% no grupo etário de 65 a 74 anos, enquanto em pessoas acima de 75 anos a incidência aumenta para $75 \%{ }^{(6)}$.

A cegueira por catarata incapacita o indivíduo, aumenta sua dependência, reduz sua condição social e resulta em aposentaria profissional precoce ${ }^{(7)}$. A única forma de reabilitar essa população é através de um procedimento cirúrgico, que pode ser realizado por diversas técnicas, sendo as mais utilizadas a facoemulsificação e a extração extracapsular do cristalino (5,8-12). $^{2}$.

A facoemulsificação (palavra derivada do grego phacos, cristalino) consiste na fragmentação e aspiração do cristalino opacificado por meio de uma pequena incisão utilizando-se energia ultrassônica e um sistema de emissão e aspiração de fluidos ${ }^{(13)}$

Do ponto de vista técnico, há inúmeros motivos que fazem da facoemulsificação a técnica mais utilizada em cirurgias de catarata no mundo, entre eles, podemos citar a menor incisão, menor trauma ao olho, maior rapidez e segurança no ato cirúrgico, além da recuperação visual ser rápida ${ }^{(9,14-18)}$.

Porém, a facoemulsificação, é mais complexa e demorada no seu aprendizado, em especial na prevenção e tratamento das possíveis complicações advindas desta complexidade tecnológica ${ }^{(19-20)}$

O ensinamento cirúrgico representa um desafio, tanto para o orientador, que leva à sala cirúrgica sua experiência e o conhecimento de como lidar com as situações inesperadas, 
quanto para o cirurgião iniciante, que está tentando aprender os passos básicos de um procedimento(20).

Desta forma, o Conselho Brasileiro de Oftalmologia (CBO), em parceria com a empresa Alcon $^{\circledR}$, criou um programa de ensino da facoemulsificação, adaptado do método de ensino usado em residências no Japão, com o propósito de capacitar os serviços de residência, melhorando sua infraestrutura e oferecendo apoio didático através da aplicação de um método de ensino padronizado.

O presente estudo tem o objetivo de analisar os resultados obtidos com a aplicação do programa de ensino de facoemulsificação CBO/ALCON no curso de especialização em oftalmologia do Hospital de Olhos do Paraná.

\section{O PROGRAMA DE ENSINO}

Como a facoemulsificação é uma cirurgia etapa dependente, os passos iniciais tem grande importância, pois problemas em sua realização trazem, frequentemente, o comprometimento de todo o procedimento.

Por essa razão, foi adotado o método "reverso", de modo que o aluno inicia seu aprendizado pelas etapas finais da cirurgia, enquanto o instrutor avança no procedimento de forma didática, utilizando a técnica de "stop and chop".

O aprendizado é dividido em três blocos: inicialmente com ensino teórico, na sequência com treinamento cirúrgico em wet lab e, finalizando, com o treinamento cirúrgico no paciente, assistido pelo instrutor. Este último bloco de treinamento consta de cinco etapas, chamadas de "check points". Após ter realizado no mínimo quatro cirurgias, de maneira satisfatória, o aluno prossegue para o próximo "check point".

As etapas são apresentadas a seguir (Tabela 1), relacionando os passos realizados pelo instrutor e pelo aluno.

\section{MÉTODOS}

Realizou-se um estudo retrospectivo analítico, com os pacientes provenientes do ambulatório do SUS do Hospital de Olhos do Paraná submetidos à cirurgia de catarata através do programa de ensino de facoemulsificação CBO/ALCON, no período compreendido entre 10 de março a 1 de dezembro de 2009.

O projeto de pesquisa deste estudo foi aprovado pelo Comitê de Ética em Pesquisa da Universidade Positivo, em 18 de março de 2010.

Participaram do programa três residentes do segundo ano do curso de especialização em oftalmologia do Hospital de Olhos do Paraná, sempre orientados pelo mesmo instrutor.

Foram incluídos todos os pacientes examinados que haviam apresentado baixa acuidade visual (BAV) progressiva devida somente à catarata, respeitando-se os critérios de inclusão e exclusão previamente estabelecidos. Os critérios de inclusão foram: pacientes que apresentavam prontuário médico completo para obtenção dos dados a serem analisados como: idade, sexo, acuidade visual (AV) pré e pós-operatória, relato da presença ou não de complicações per ou pós-operatórias e em qual momento da cirurgia elas ocorreram. A catarata deveria apresentar algumas características específicas como: ser do tipo nuclear, de densidade de duas a três cruzes, com subcapsular associada somente quando esta não prejudicasse de forma significativa a visualização do reflexo vermelho e que não apresentasse extensos pontos de aderência à cápsula posterior.

Os critérios de exclusão foram: pacientes com prontuários incompletos, que não retornaram para refração pós-operatória ou com comorbidades anatomofuncionais prévias à cirurgia.

Após a cirurgia, os pacientes que não apresentaram intercorrências eram avaliados em consulta ambulatorial nos dias $1^{\circ}, 7^{\circ}$ e e $30^{\circ}$ de pós-operatório, quando então eram submetidos à refração final e recebiam alta. Aqueles que apresentaram in-

Tabela 1. Passos realizados pelo instrutor e pelo aluno em cada etapa

\begin{tabular}{|c|c|c|c|c|c|c|c|c|c|c|c|}
\hline & \multirow[b]{2}{*}{ Passos de cada etapa } & \multicolumn{5}{|c|}{$\begin{array}{c}\text { Instrutor } \\
\text { Check points }\end{array}$} & \multicolumn{5}{|c|}{$\begin{array}{l}\text { Aluno } \\
\text { Check points }\end{array}$} \\
\hline & & 1 & 2 & 3 & 4 & $\overline{5}$ & 1 & 2 & 3 & 4 & 5 \\
\hline 1) Incisões & $\begin{array}{l}\text { 1.1 Paracentese } 15^{\circ} \\
1.2 \text { Incisão límbica de } 2,75 \text { mm }\end{array}$ & $\dot{\bullet}$ & & $\stackrel{\bullet}{\bullet}$ & $\dot{\bullet}$ & - & & & & & • \\
\hline 2) Injeção de viscoelástico & & • & - & $\bullet$ & • & - & & & & & \\
\hline 3) Capsulorrexe & $\begin{array}{l}\text { 3.1 Abertura e início } \\
\text { 3.2 Volta intermediária } \\
\text { 3.3 Finalização }\end{array}$ & $\begin{array}{l}\bullet \\
\bullet \\
\bullet\end{array}$ & - & - & • & & & - & • & • & $\begin{array}{l}\bullet \\
\bullet \\
\bullet\end{array}$ \\
\hline 4) Hidrodissecção e hidrodelineação & & - & • & $\bullet$ & & & & & & • & • \\
\hline \multirow[t]{4}{*}{ 5) Stop and chop do núcleo do cristalino } & 5.1 Sulco & & & & & & & & & & \\
\hline & $\begin{array}{l}\text { 5.1.1 Confecção de metade do sulco } \\
\text { 5.1.2 Rotação do núcleo } \\
\text { 5.1.3 Finalização do sulco }\end{array}$ & $\begin{array}{l}\bullet \\
\bullet \\
\bullet\end{array}$ & $\begin{array}{l}\bullet \\
\bullet \\
\bullet\end{array}$ & & & & & & $\begin{array}{l}\bullet \\
\bullet \\
\bullet\end{array}$ & $\begin{array}{l}\bullet \\
\bullet \\
\bullet\end{array}$ & $\begin{array}{l}\bullet \\
\bullet \\
\bullet\end{array}$ \\
\hline & $\begin{array}{l}\text { 5.2 Fraturas } \\
\text { 5.2.1 Fratura do sulco } \\
\text { 5.2.2 Fratura (chop) do primeiro heminúcleo } \\
\text { 5.2.3 Fratura (chop) do segundo heminúcleo }\end{array}$ & $\begin{array}{l}\bullet \\
\bullet \\
\bullet\end{array}$ & $\stackrel{\bullet}{\bullet}$ & • & & & & & $\begin{array}{l}\bullet \\
\bullet\end{array}$ & $\begin{array}{l}\bullet \\
\bullet \\
\bullet\end{array}$ & $\begin{array}{l}\bullet \\
\bullet \\
\bullet\end{array}$ \\
\hline & $\begin{array}{l}\text { 5.3.1 Conquista do primeiro quadrante } \\
\text { 5.3.2 Conquista do segundo quadrante }\end{array}$ & $\dot{\bullet}$ & $\bullet$ & • & • & & & $\cdot$ & - & $\bullet$ & $\dot{\bullet}$ \\
\hline \multicolumn{2}{|c|}{ 6) Irrigação e aspiração de remanescentes corticais } & $\cdot$ & & & & & & $\cdot$ & $\cdot$ & • & $\cdot$ \\
\hline \multicolumn{2}{|c|}{ 7) Injeção de viscoelástico no saco capsular e na câmara anterior } & & & & & & $\cdot$ & $\cdot$ & • & • & $\bullet$ \\
\hline \multicolumn{2}{|c|}{ 8) Inserção da LIO dobrável com auxílio de um injetor } & & & & & & $\cdot$ & $\cdot$ & • & • & $\bullet$ \\
\hline \multicolumn{2}{|c|}{ 9) Aspiração de viscoelástico } & & & & & & $\cdot$ & $\bullet$ & • & • & $\bullet$ \\
\hline \multicolumn{2}{|l|}{ 10) Sutura com fio nylon 10.0} & & & & & & • & $\bullet$ & • & $\bullet$ & - \\
\hline
\end{tabular}


tercorrências, tanto no per quanto no pós-operatório, foram avaliados com maior frequência, conforme a evolução de cada caso.

As cirurgias foram realizadas com o aparelho de facoemulsificação ALCON LEGACY Everest ${ }^{\circledR}$. As lentes intraoculares $(\mathrm{LIO})$ utilizadas eram de silicone dobráveis, de $6,0 \mathrm{~mm}$ de diâmetro, ou dobráveis de acrílico hidrofóbico de $6,0 \mathrm{~mm}$ de diâmetro, e o viscoelástico utilizado foi a metilcelulose $2 \%$.

Concluído o processo de aprendizagem dos residentes e finalizados os atendimentos dos pacientes que participaram do projeto, os dados selecionados foram agrupados e analisados.

\section{RESULTADOS}

Dos 100 prontuários selecionados, foram incluídos 68 pacientes (84 olhos), de acordo com os critérios de inclusão e exclusão. Os 32 prontuários restantes foram excluídos devido à falta de dados suficientes para o trabalho ou por serem pacientes portadores de comorbidades como diabetes e glaucoma. A idade dos pacientes variou de 30 a 85 (média de 67,9 $\pm 13,6$ anos), sendo $28(41,18 \%)$ do sexo masculino e $40(58,82 \%)$ do sexo feminino.

A melhor acuidade visual, corrigida, no período pré-operatório, variou de visão de conta dedos a 20/30, e no pósoperatório, de 20/200 a 20/20 (Gráfico 1).

Do total de 84 olhos operados, 77 (91,67\%) apresentaram acuidade visual (AV) final de 20/40 ou melhor. Entre as intercorrências, a principal delas foi a rotura de cápsula posterior (RCP) e ocorreu em 11 (13,09\%) dos 13 (15,48\%) olhos que apresentaram algum tipo de complicação. Ocorreu RCP em 2 olhos (2,38\%) no "check point"1, 2 olhos (2,38\%) no "check point" 2, 2 olhos (2,38\%) no "check point" 3, 1 olho $(1,19 \%)$ no "check point" 4 e 4 olhos (4,76\%) no "check point" 5 (Gráfico 2).

Dentre os casos em que a acuidade visual final pós-operatória foi igual ou pior que 20/60, totalizando 7 olhos (8,33\%), 3 olhos apresentaram rotura de cápsula posterior como única intercorrência; 1 olho apresentou rotura de cápsula posterior seguido de endoftalmite no segundo dia de pós-operatório, sendo o paciente submetido à vitrectomia via pars plana e antibioticoterapia intravítrea após coleta de material para

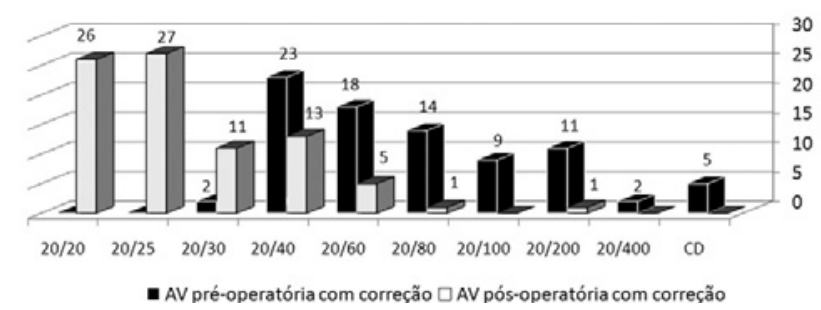

Gráfico 1 . Estratificação da AV pré e pós-operatória com melhor correção óptica.

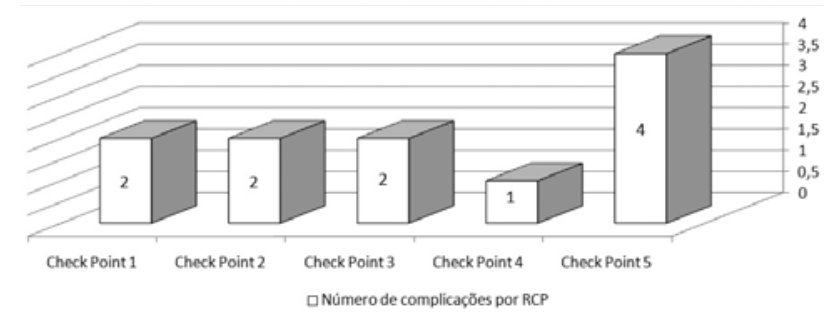

Gráfico 2 . Número de complicações por rotura de cápsula posterior em cada check point. cultura, apresentando Staphylococus aureus como patógeno, evoluindo com acuidade visual final de 20/60; 1 olho apresentou no pós-operatório tardio opacidade difusa de córnea (20/60); 1 olho apresentou rotura de cápsula posterior com luxação de fragmento cortical do cristalino na câmara vítrea, obtendo acuidade visual final de 20/80 e 1 olho apresentou rotura de cápsula posterior com luxação do núcleo cristalino na câmara vítrea, sendo submetido à vitrectomia via pars plana posteriormente, evoluindo com acuidade visual final de 20/200.

Dentre outras intercorrências, 1 olho apresentou erro de cálculo do grau da lente intraocular à biometria devido à presença de estafiloma posterior, sendo submetido ao novo cálculo biométrico e troca da lente intraocular, evoluindo com acuidade visual final de 20/40; 1 olho apresentou glaucoma inflamatório pós-operatório, sendo tratado clinicamente, obtendo controle satisfatório da pressão intraocular, evoluindo com acuidade visual final de 20/25.

A tabela 2 mostra a quantidade de intercorrências intra e pós-operatórias, assim como as concomitâncias de eventos que se apresentaram em um mesmo olho.

\section{DISCUSSÃO}

A cirurgia de catarata é o procedimento cirúrgico oftalmológico mais comumente realizado no Brasil, sendo a facoemulsificação a técnica mais utilizada devido a diversos fatores como possibilidade de microincisão tunelizada com aplicação de lente intraocular (LIO) dobrável (diminuindo astigmatismo induzido), menor tempo cirúrgico e dano corneoendotelial, possibilitando uma recuperação pós-operatória mais rápida ${ }^{(1-4)}$. Portanto, um treinamento qualificado da técnica deve ser um dos principais objetivos de qualquer programa de residência de oftalmologia. Por ser uma cirurgia passo-dependente, foi adotado neste programa de ensino proposto pelo $\mathrm{CBO}$, o método "reverso".

Os possíveis benefícios de se ensinar pelo método "reverso" incluem: aplicação de um método de ensino padronizado, redução do número de complicações nas etapas iniciais do procedimento cirúrgico e, consequentemente, o paciente apresentar um melhor resultado.

Neste estudo, as cirurgias foram divididas em cinco "check points", de acordo com os passos cirúrgicos, sempre na tentativa de diminuir o número de complicações. Observou-se um menor número de intercorrências per-operatórias nos primeiros "check points" (1 ao 4) em relação ao último ${ }^{(5)}$. Embora o número de intercorrências tenha sido maior, não existem dados para afirmar que as RCPs ocorridas no último "check point" tiveram pior evolução, mas provavelmente ocorreram porque o aluno já realizava todos os passos da cirurgia.

Ainda não há publicado nenhum estudo com a aplicação do método de ensino "reverso" para compararmos aos nossos resultados, mas alguns estudos demonstraram as complicações ocorridas em relação ao tempo de aprendizado.

Em 1998, alguns autores(21) apresentaram a redução estatisticamente significativa do número de complicações cirúrgicas nas 50 últimas cirurgias de facoemulsificação realizadas por residentes quando comparadas com as 50 primeiras. Enquanto, no estudo de outros autores ${ }^{(22)}$ não houve diferença estatisticamente significativa entre as complicações per-operatórias nas primeiras e últimas cirurgias.

Várias complicações per-operatórias podem ocorrer no aprendizado da facoemulsificação, sendo a rotura de cápsula posterior (RCP) a mais frequente ${ }^{(14-15,23-24)}$.

A tabela 3 mostra os resultados dos principais estudos que comparam a porcentagem de rotura de cápsula posterior nos 
Tabela 2. Relação de intercorrências intra e pós-operatórias

\begin{tabular}{lcc}
\hline Olho & Intra-operatória & Pós-operatória \\
\hline 1 & $R C P$ & Endoftalmite \\
2 & $R C P$ & Opacidade difusa da córnea \\
3 & $R C P+$ luxação de fragmento na câmara vítrea & - \\
4 & $R C P+$ luxação do núcleo na câmara vítrea & - \\
5 & - & Erro de biometria \\
6 & - & Glaucoma inflamatório \\
$7,8,9,10,11,12$ e 13 & $R C P$ & - \\
\hline$* R C P=$ rotura de cápsula posterior & &
\end{tabular}

Tabela 3. Porcentagem de rotura de cápsula nos olhos submetidos à facoemulsificação, em diferentes trabalhos publicados

\begin{tabular}{lccc}
\hline Autor & Ano & No de olhos & RCP* No (\%) \\
\hline Dantas et al. ${ }^{(24)}$ & 1995 & 50 & $12(18,0)$ \\
Tarbet et al. ${ }^{(25)}$ & 1995 & 300 & $17(5,6)$ \\
Lambert et al. ${ }^{(14)}$ & 1997 & 48 & $5(10,0)$ \\
Robin et al. ${ }^{(26)}$ & 1998 & 300 & $40(13,3)$ \\
Prasad et al.(27) & 1998 & 102 & $6(5,6)$ \\
Neustein et al. ${ }^{(28)}$ & 1998 & 100 & $13(14,1)$ \\
Estudo atual & 2009 & 84 & $11(13,09)$ \\
\hline
\end{tabular}

${ }^{*} \mathrm{RCP}=$ rotura de cápsula posterior

olhos submetidos à facoemulsificação. Neste estudo, a porcentagem de RCP $(13,09 \%)$ ficou dentro da média em comparação a outros trabalhos publicados ${ }^{(14,25-28)}$.

Nos "check points" iniciais (1 e 2), as roturas de cápsula posterior ocorreram provavelmente devido ao implante da LIO de silicone, através de injetor, que apresentava uma abertura muito brusca dentro da câmara anterior.

O único caso de endoftalmite ocorreu devido a um instrumento cirúrgico contaminado, o qual foi enviado para cultura, sendo encontrado Staphylococcus aureus em sua superfície, assim como encontrado na cultura do vítreo coletado do paciente.

Os resultados deste estudo corroboram com uma tendência atual de que o ensino de facoemulsificação deva ser encorajado para residentes com pouca ou nenhuma experiência com técnica de extração extracapsular do cristalino (EEC), pelo método "reverso", com supervisão de um cirurgião experiente.

\section{CONCLUSÃO}

O método "reverso" proposto para o ensino de facoemulsificação pelo programa CBO/ALCON se mostra uma forma de ensino adequada, para o médico residente e seu instrutor, e principalmente para o paciente, devido ao baixo índice de complicações.

\section{REFERÊNCIAS}

1. Martorell Alemañy J, Villar Valdés R. Oftalmología. La Habana: Editorial Ciências Médicas; 2003

2. West S. Epidemiology of cataract: accomplishments over 25 years and future directions. Ophthalmic Epidemiol. 2007;14(4):173-8.

3. Rutzen AR, Ellish NJ, Schwab L, Graham PJ, Pizzarello LD, Hemady RK, Maldonado $\mathrm{MJ}$; Cambodia Eye Survey Group. Blindness and eye disease in Cambodia. Ophthalmic Epidemiol. 2007;14(6):360-6.

4. Tabin G, Chen M, Espandar L. Cataract surgery for the developing world. Curr Opin Ophthalmol. 2008;19(1):55-9.

5. Snellingen T, Evans JR, Ravilla T, Foster A. Surgical interventions forage-related cataract. Cochrane Database Syst Rev 2002;(2):CD001323. Update in: Cochrane Database Syst Rev. 2006;(4):CD001323.
6. American Academy of Ophthalmology. Cataract in the otherwise healthy adult eye. San Francisco: AAO/ Preferred Practice Pattern; 1989.

7. Kara-José Junior N, Avakian A, Lower LMT, Rocha AM, Cursino M, Alves MR. Facoemulsificação versus extração extracapsular manual do cristalino: análise de custos. Arq Bras Oftalmol. 2004;67(3):481-9.

8. Tronca GA, Netto AA, Nassar SM. Prognóstico visual na cirurgia de facoemulsificação da catarata com implante de lente intraocular. Arq Catarin Med. 2005;34(2): 39-48.

9. Minassian DC, Rosen P, Dart JK, Reidy A, Desai P, Sidhu M, et al. Extracapsular cataract extraction compared with small incision surgery by phacoemulsification: a randomised trial. Br J Ophthalmol. 2001;85(7):822-9. Erratum in: Br J Ophthalmol. 2001;85(12):1498. Comment in: Br J Ophthalmol. 2001;85(7):1498.

10. Ohrloff C, Zubcov AA. Comparison of phacoemulsification and planned extracapsular extraction. Ophthalmologica. 1997;211(1):8-12

11. Albanis C, Dwyer MA, Ernest JT. Outcomes of extra-capsular cataract extraction and phacoemulsification performed in a university training program. Ophthalmic Surg Lasers. 1998;29(8):643-8.

12. Leinonen J, Laatikainen L. Changes in visual acuity of patietns undergoing cataract surgery during the last two decades. Acta Ophthalmol Scand. 2002:80(5):506-11.

13. Monteiro, JV. O presente e o futuro da cirurgia de catarata. Universo Visual: a revista da oftalmologia [Internet]. 2010 [citado 2009 abr 23];52 [1 p.] Disponível em:. http:/ /www.universovisual.com.br/publisher/preview.php?id_mat=210

14. Lambert LC, Occhiutto ML, Paparelli CM, Kniggendorf S, Akaishi L, Mendonça BD et al. Resultados visuais e incidência de complicações em facoemulsificação com LIO por residentes. Rev Brás Oftalmol. 1997;56(12):953-6.

15. Rodrigues Martín J, Baudet Naveros B, Martin Barrera F, Abreu Reyes JA. Cirurgía de la catarata: de la extracción extracapsular a la facoemulsificação. Evolución y resultados obtenidos por un residente de tercer año. Arch Soc Canar Oftalmol. 2000;11(1):1-4.

16. Domingues FGP, Crema AS, Yamane Y. Complicações intra-operatórias da facoemulsificação durante a residência médica. Rev Bras Oftalmol. 2000;59(4):275-9.

17. Leaming DV. Practice styles and preferences os ASCRS members - 1998 survey. J Cataract Refract Surg. 1999;25(6):851-9.

18. The Royal College of Ophthalmologists. Cataract surgery guidelines. London: Royal College of Ophthalmologists; Feb. 2001.21 p. [cited 2003 Nov 20]. Available from: http://www.rcophth.ac.uk/publications/guidelines/cataract_surgery2.html

19. Nordlund ML, Marques DM, Marques FF, Cionni RJ, Osher RH. Techniques for managing common complications of cataract surgery. Curr Opin Ophthalmol. 2003;14(1):7-19.

20. Ghanem VC, Mannis MJ. O professor e o estudante na facoemulsificação: os dez princípios para o sucesso. Arq Bras Oftalmol. 2003;66(1):93-9.

21. Corey RP, Olson RJ. Surgical outcomes of cataract extractions performed by residents using phacoemulsification. J Cataract Refract Surg. 1998;24(1):66-72. Comment in: J Cataract Refract Surg. 1998.24(6):727-9.

22. Centurion V, Falvo SA, Caballero JC, Lacava AC. Complicações durante a facoemulsificação: as primeiras 500 vs as últimas cirurgias após 10 anos de experiência. Rev Bras Oftalmol. 2002:61(9):651-6.

23. Cruz OA, Wallace GW, Gay CA, Matoba AY, Koch DD. Visual results and complications of phacoemulsification with intraocular lens implantation performed by ophthalmology residents. Ophthalmology. 1992;99(3):448-52. Comment in: Ophthalmology. 1992;99(8):1181.

24. Dantas PE, Dantas MC, Mandia Jr C, Waiswol M, Krasilchik G, Dias AK. Facoemulsificação: a experiência da conversão - Análise dos primeiros casos. Arq Bras Oftalmol. 1995;58(6):421-4.25. Tarbet KJ, Mamalis N, Theurer J, Jones BD, Olson RJ. Complications and results of phacoemulsification performed by residents. J Cataract Refract Surg. 1995;21(6):661-5.

26. Robin AL, Smith S, Natchiar G, Ramakrishnan R, Srinivasan, Raheem R, et al. The initial complication rate of phacoemulsification in India. Invest Ophthalmol Vis Sci. 1997;38(11):2331-7

27. Prasad S. Phacoemulsification learning curve: experience of two junior trainee ophthalmologists. J Cataract Refract Surg. 1998;24(1):73-7.

28. Neustein I, Araújo ME, Chou AC, Silva CR, Oliveira LB. Facoemulsificação: resultados e complicações nos primeiros 100 olhos. Arq Bras Oftalmol. 2000;63(1):29-31. 